\title{
Ground Plane Motion Parameter Estimation For Non Circular Paths
}

\author{
G.J.Ellwood Y.Zheng S.A.Billings \\ Department of Automatic Control and Systems Engineering \\ University of Sheffield, Sheffield, UK \\ J.E.W.Mayhew J.P.Frisby \\ Artificial Intelligence Vision Research Unit, \\ University of Sheffield, Sheffield, UK
}

\begin{abstract}
A method of motion parameter estimation for AGV's is developed based on a new trajectory constraint algorithm. It is assumed that the vehicle will follow a circular path over the vision sampling interval. The algorithm has been found to be more effective and consistent than least squares estimators when the motion obeys the trajectory constraint. Reliable estimates of the motion parameters can even be made from individual data pairs. In this way, a static world can be segmented from moving objects, and so the motion parameters can be obtained using the stationary points alone.

In practice the vehicle will not necessarily follow a circular path, and hence there may be a bias in the parameter estimates. Experiments were carried out using simulated data, where the true trajectory was a clothoid, to investigate the robustness of the algorithm when the trajectory constraint is violated.
\end{abstract}

\section{Motion Parameter Recovery}

The prime objective of the work is to estimate the rotation and translation parameters of a vehicle using a pair of stereo images. Corners are found in the images using the Plessey group algorithm [1]. These are matched to form a 3D point based map of the robot's environment [6]. The vehicle then moves in the ground plane. The procedure is repeated for the new coordinate frame of the AGV.

A linked list of the corners $\mathrm{q}_{\mathbf{i}}$ observed at time $t$ and their corresponding coordinates $\mathbf{p}_{\mathbf{i}}$ observed at $t+1$ is formed.

The motion parameters $R$ and $T$ are found by solution of the equation

$$
\mathbf{q}_{\mathbf{i}}=R \mathbf{p}_{\mathbf{i}}+T
$$

Most algorithms achieve this using the principle of least squares to estimate the rotation matrix $R$. The translation vector $T$ is then obtained from equation(1).

It has been observed by many authors $[3,5]$ that although the vehicle moves smoothly, the estimated paths are very erratic and noisy. Such trajectories are impossible. This is due to the sensitivity of least squares methods in general to 
noise, causing the estimate of $R$ to be significantly degraded. This is magnified when solving for $T$ using equation (1). The points $\mathbf{q}_{\mathbf{i}}$ and $\mathbf{p}_{\mathbf{i}}$ are usually distant from the vehicle, so a small error in $R$ will be amplified through to yield a large error in $T$.

Our proposal was to constrain the parameters by some assumptions [5] regarding the dynamics of the vehicle. A clear constraint on the motion would be that, if it does not skid, the vehicle will always move in the direction it is heading. It seems reasonable to assume also that the rate of change of steering is small. So, provided the vision sampling rate is sufficiently fast, one can assume that the rate of change of steering is approximately zero over the sampling interval. This means the vehicle is travelling along a path of constant curvature, or a circle, between samples.

A geometrical analysis [5] shows that, when moving along a circular arc, the three motion parameters can be related by

$$
t_{x}=t_{z} \tan \frac{\theta}{2}
$$

Expanding equation (1), there are three equations and three unknowns. The parameters can be found analytically.

This method has been found to be a considerable improvement on least squares in the estimation of lateral motion and rotation for motion obeying the circular constraint. There is little change in the accuracy of the depth motion parameter estimate.

The motion parameters can be estimated from each individual data pair. This property can be used to segment [4] moving objects from a static background. The estimates made using stationary points will form a cluster in the motion parameter space. The corners on an object moving arbitrarily in the scene will not in general satisfy the trajectory constraint. The motion parameters obtained using these points will be scattered widely in the motion parameter space.

Each of the motion parameters are arranged in ascending order of the heading parameter $\theta$. A threshold based on the variance of $\theta$ is set up such that if two adjacent motion parameters in the ordered list represent the vehicle's motion, their difference in heading is almost certainly below the threshold. Another threshold $L_{t h}$ is set up on the minimum length of a group. This is to avoid groupings between consecutive points which coincidentally have similar headings rather than because they are coherent. If both $\theta_{t h}$ and $L_{t h}$ are set up appropriately, only one cluster should be obtained with members representing the motion parameters calculated from the static world.

For a vehicle to follow a planned trajectory, obeying the trajectory constraint, would require instananeous steering at the moment of sampling. This is, of course, impossible. There will, therefore, always be a bias in the estimates of the motion parameters. It is necessary therefore to investigate the effect that violation of this assumption has upon parameter estimation and scene segmentation. 


\section{Simulated Experiments}

\subsection{Trajectory Generation}

A series of experiments were carried out in which the "true" motion of the vehicle was a clothoid. This a function [2] whose curvature $c$ varies linearly with respect to the distance $s$ moved along it.

$$
c(s)=k s+c_{0}
$$

where $k$ is known as the sharpness coefficient. The direction of heading is therefore

$$
\theta(s)=\int_{0}^{s}\left(k s+c_{0}\right) d s=\frac{k s^{2}}{2}+c_{0} s+\theta_{0}
$$

A set of clothoids is illustrated in figure (1). The larger the coefficient $k$, the tighter will be the curve.

The parameter $k$ can be thought of as representing, physically, the rate of steering. It is however quite hard to grasp what any particular value of $k$ will mean.

A commonly desired trajectory would be to join two straight lines at right angles to each other. A solution is a clothoid pair. This is a trajectory that starts with a coefficient $k$ until a turn of $45^{\circ}$ is achieved, it then continues with sharpness $-k$ until the total change in heading is $90^{\circ}$. The distance $t_{z}$ moved to join $P_{0}$ to $P_{1}$ for a certain sharpness $k$ gives a better intuitive grasp of the sharpness of the curve than the value of $k$ itself.

It is noted that a clothoid will not in reality be the true trajectory. It is used here, simply because it represents a trajectory that does not obey the constraint of a constant curvature.

\subsection{Data Generation}

At time $t$, a set of 3D points were generated in front of the vehicle. All the coordinates were generated using independent Gaussian distributions.

Assume that at time $t+1$ the vehicle has moved to a new location. The motion parameters were calculated. Using these parameters, the points generated at time $t$ were mapped onto the vehicles new local coordinate frame at time $t+1$. Each point at $t+1$ had a known correspondence with a point at time $t$. Points which could not be seen by the vehicle at both times were rejected. Data were generated until a predetermined number, in this case 20 , were visible to the vehicle before and after motion.

The two sets of points were then transformed into left and right camera images for times $t$ and $t+1$, using the exact calibration file. Each image was then blurred to produce two sets of 3D data points, with known correspondences. The motion parameters were then estimated using both the trajectory constraint and the least squares algorithms. 


\subsection{Experimental Results}

\subsubsection{The Effect of Violating the Trajectory Constraint on Estimating Motion Parameters}

Experiments were carried out to investigate the effect of violating the trajectory constraint on parameter estimation. In each of the experiments the vehicle moved a distance of $250 \mathrm{~mm}$ along the curve. This is equivalent to moving at $1.25 \mathrm{~m} / \mathrm{s}$ with a vision sampling rate of $5 \mathrm{~Hz}$

The vehicle turned through $5.73^{\circ}$ over the sampling interval, following several trajectories. These had sharpness coefficients (see equation (3)) of 0 (ie a circle), $1 \times 10^{-6}, 2 \times 10^{-6}, 3 \times 10^{-6}$ and $4 \times 10^{-6}$. Clothoid pairs of such sharpness would join two perpendicular straight lines in $\infty \mathrm{m}, 1.5 \mathrm{~m}, 1.2 \mathrm{~m}, 1.0 \mathrm{~m}$ and $0.8 \mathrm{~m}$ respectively. Clothoids with corresponding positive sharpness coefficients are illustrated in figure 1 . Estimates of the bias and standard error were made using 50 identical samples of each movement.

The bias and standard error for the parameter estimates for these experiments can be seen in Tables 1 to 3 . The results of an individual experiment where $k=4 \times 10^{-6}$ can be seen in figures 2,3 and 4 .

The estimates obtained using least squares appear to be unbiased, but consistently noisier than those obtained using the trajectory constraint method.

It is noted that there is a bias in the estimates of $\theta$ (see table 1 and figure 2) for the trajectory constraint method. This increases with the degree to which the circular assumption is violated and arises because equation (2) is no longer valid. For motion along a clothoid trajectory the direction of heading is given by

$$
\theta(s)=\frac{k s^{2}}{2}+c_{0} s
$$

The Trajectory Constraint Method assumes that $\theta$ is given by

$$
\theta(s)_{e s t}=c^{\prime} s
$$

where $c^{\prime}$ is some constant value. Therefore, the bias is given by

$$
\left(\theta-\theta_{e s t}\right)=\frac{k s^{2}}{2}+\left(c_{0}-c^{\prime}\right) s
$$

One would therefore expect the bias to be linear with respect to $k$ This is shown in figure 5 .

Results for estimating the depth parameter can be seen in table 2 and figure 3. There was little difference in the estimates of $t_{z}$ for either technique. For small $\theta$, the error in $t_{z}$ is given by

$$
\tilde{t}_{z} \approx\left(c_{p x}+c_{p z} \theta\right) \tilde{\theta}+\tilde{c}_{q z}+\tilde{c}_{p x} \theta-\tilde{c}_{p z}
$$

The bias is caused by the terms in $\tilde{\theta}$, but these are small, of the order $10^{-1}$. $\tilde{c}_{q z}$ and $\tilde{c}_{p z}$ will be of the order $10^{1}$ to $10^{2}$ and since the error in each estimate will be dominated by these terms. $t_{z}$ is effectively unbiased.

Any improvement in the estimates of $t_{x}$ and $\theta$ will have little effect on the $t_{z}$ estimate. The accuracy of $t_{z}$ will only will be significantly improved by better error modelling of the vision data. 


\begin{tabular}{|c|c|c|c|c|c|}
\hline & $\theta^{\circ}$ & \multicolumn{2}{|c|}{ LS2D } & \multicolumn{2}{c|}{ traj } \\
\hline Sharpness & true & bias & sd & bias & sd \\
\hline 0 & 5.73 & -0.01 & 0.08 & 0.0005 & 0.008 \\
$1 \times 10^{-6}$ & 5.73 & -0.005 & 0.039 & -0.043 & 0.013 \\
$2 \times 10^{-6}$ & 5.73 & 0.0037 & 0.062 & -0.077 & 0.016 \\
$3 \times 10^{-6}$ & 5.73 & 0.0039 & 0.065 & -0.122 & 0.023 \\
$4 \times 10^{-6}$ & 5.73 & 0.014 & 0.063 & -0.159 & 0.029 \\
\hline
\end{tabular}

Table 1: Bias and Standard Deviation for Estimates of $\theta$ for Increasing Violation of Trajectory Constraint, $\theta_{\text {true }} \approx 0.1 \mathrm{rad}$

\begin{tabular}{|c|c|c|c|c|c|}
\hline & $t_{z}(\mathrm{~mm})$ & \multicolumn{2}{|c|}{ LS2D } & \multicolumn{2}{c|}{ traj } \\
\hline Sharpness & true & bias & sd & bias & sd \\
\hline 0 & 249.6 & 0.78 & 2.27 & 0.74 & 2.17 \\
$1 \times 10^{-6}$ & 249.7 & 0.85 & 2.46 & 0.90 & 2.48 \\
$2 \times 10^{-6}$ & 249.8 & -0.33 & 2.57 & -0.36 & 2.69 \\
$3 \times 10^{-6}$ & 249.8 & -0.59 & 2.37 & -0.60 & 2.46 \\
$4 \times 10^{-6}$ & 249.9 & 0.03 & 2.73 & -0.17 & 2.85 \\
\hline
\end{tabular}

Table 2: Bias and Standard Deviation for Estimates of $t_{z}$ for Increasing Violation of Trajectory Constraint, $\theta_{\text {true }} \approx 0.1 \mathrm{rad}$

\begin{tabular}{|c|c|c|c|c|c|}
\hline & $t_{x}(\mathrm{~mm})$ & \multicolumn{2}{c|}{ LS2D } & \multicolumn{2}{c|}{ traj } \\
\hline Sharpness & true & bias & sd & bias & sd \\
\hline 0 & 12.50 & 0.29 & 2.97 & 0.005 & 0.11 \\
$1 \times 10^{-6}$ & 11.20 & 0.20 & 1.33 & 1.21 & 0.098 \\
$2 \times 10^{-6}$ & 9.90 & -0.097 & 2.58 & 2.43 & 0.11 \\
$3 \times 10^{-6}$ & 8.60 & -0.15 & 2.55 & 3.64 & 0.13 \\
$4 \times 10^{-6}$ & 7.30 & -0.58 & 2.47 & 4.87 & 0.14 \\
\hline
\end{tabular}

Table 3: Bias and Standard Deviation for Estimates of $t_{x}$ for Increasing Violation of Trajectory Constraint, $\theta_{\text {true }} \approx 0.1 \mathrm{rad}$

\begin{tabular}{|c|c|c|c|c|c|}
\hline & $\theta^{\circ}$ & \multicolumn{2}{|c|}{ LS2D } & \multicolumn{2}{c|}{ traj } \\
\hline Sharpness & true & bias & sd & bias & sd \\
\hline 0 & 16.12 & -0.0003 & 0.05 & 0.0008 & 0.0043 \\
$1 \times 10^{-6}$ & 16.12 & 0.004 & 0.057 & -0.030 & 0.0059 \\
$2 \times 10^{-6}$ & 16.12 & -0.0019 & 0.068 & -0.062 & 0.0072 \\
$3 \times 10^{-6}$ & 16.12 & 0.0039 & 0.046 & -0.095 & 0.0078 \\
$4 \times 10^{-6}$ & 16.12 & -0.009 & 0.044 & -0.13 & 0.010 \\
\hline
\end{tabular}

Table 4: Bias and Standard Deviation for Estimates of $\theta$ for Increasing Violation of Trajectory Constraint, $\theta_{\text {true }} \approx 0.281 \mathrm{rad}$

\begin{tabular}{|c|c|c|c|c|c|}
\hline & $t_{x}(\mathrm{~mm})$ & \multicolumn{2}{|c|}{ LS2D } & \multicolumn{2}{c|}{ traj } \\
\hline Sharpness & true & bias & sd & bias & sd \\
\hline 0 & 34.96 & -0.12 & 2.17 & -0.13 & 0.37 \\
$1 \times 10^{-6}$ & 33.67 & -0.18 & 2.45 & 1.23 & 0.47 \\
$2 \times 10^{-6}$ & 32.39 & 0.20 & 3.07 & 2.51 & 0.47 \\
$3 \times 10^{-6}$ & 31.10 & -0.07 & 2.06 & 3.73 & 0.38 \\
$4 \times 10^{-6}$ & 29.80 & 0.37 & 2.05 & 4.83 & 0.34 \\
\hline
\end{tabular}

Table 5: Bias and Standard Deviation for Estimates of $t_{x}$ for Increasing Violation of Trajectory Constraint, $\theta_{\text {true }} \approx 0.281 \mathrm{rad}$ 


\begin{tabular}{|c|c|c|c|c|c|}
\hline & $\theta^{o}$ & \multicolumn{2}{|c|}{ LS2D } & \multicolumn{2}{c|}{ traj } \\
\hline Distance & true & bias & sd & bias & sd \\
\hline 100 & 16.11 & 0.004 & 0.03 & -0.005 & 0.004 \\
200 & 16.11 & 0.0062 & 0.032 & -0.033 & 0.004 \\
300 & 16.10 & 0.0008 & 0.06 & -0.11 & 0.01 \\
400 & 16.10 & 0.001 & 0.037 & -0.26 & 0.021 \\
\hline
\end{tabular}

Table 6: Bias and Standard Deviation for Estimates of $\theta$ for Increasing Distance Moved over Sampling Interval, $\theta_{\text {true }} \approx 0.281 \mathrm{rad}$

\begin{tabular}{|c|c|c|c|c|c|}
\hline & $t_{x} m m$ & \multicolumn{2}{|c|}{ LS2D } & \multicolumn{2}{c|}{ traj } \\
\hline Distance & true & bias & sd & bias & sd \\
\hline 100 & 13.82 & -0.20 & 1.43 & 0.13 & 0.40 \\
200 & 26.62 & -0.21 & 1.41 & 1.36 & 0.42 \\
300 & 37.45 & -0.21 & 2.56 & 4.02 & 0.34 \\
400 & 45.30 & -0.016 & 1.56 & 9.68 & 0.39 \\
\hline
\end{tabular}

Table 7: Bias and Standard Deviation for Estimates of $t_{x}$ for Increasing Distance Moved over Sampling Interval, $\theta_{\text {true }} \approx 0.281 \mathrm{rad}$

The estimation of the lateral motion parameter was then examined. The results can be seen in table 3 and figure 4 . The estimates made using the trajectory constraint method were biased. Again though, they were smoother than those made using least squares. For small $\theta$, the error in $t_{x}$ is given by

$$
\tilde{t}_{x}=\tilde{c}_{q x}-\tilde{c}_{p x}-\tilde{c}_{p z} \theta-c_{q z} \tilde{\theta}
$$

The only term with bias is $\tilde{\theta}$, therefore the bias in $t_{x}$ is given by $c_{p z} \tilde{\theta}$. The bias in the estimates of lateral motion is therefore linear with respect to the bias in $\theta$. This is illustrated in figure 6 . The bias appears large with respect to $t_{x}$. It is however small with respect to the total distance travelled over the sampling interval. When viewed in this context the bias is very small.

A similar set of experiments was carried out for a rotation of approximately $16^{\circ}$ over the same sampling interval as before. The bias and standard deviation for both methods of estimation can be seen in tables 4 and 5 . The Least Squares estimates are more noisy but unbiased. There seems to be little change in the accuracy in the estimates obtained for the trajectory constraint method for the larger rotation, although the absolute value of the parameters has changed. The bias in terms of the total distance moved is the same as for the smaller turn. This suggests that the bias in $\theta$ and $t_{x}$ is a function of the degree of violation and sampling rate alone.

\subsubsection{The Effect of Sampling Rate on Parameter Estimation for Motion Violating the Trajectory Constraint}

In these experiments a clothoid of sharpness $2 \times 10^{-6}$ was used. The vehicle turned through approximately $16^{\circ}$ over the sampling interval. In each experiment the total distance $s$ moved over a sampling interval was different. As $s=V t$, this investigates the effect of the sampling rate and vehicle speed on parameter estimation. 
The results for $\theta$ and lateral motion can be seen in tables 6 and 7 . The least squares estimates seem to be unaffected by the sampling interval. However, the bias in the estimates obtained using the trajectory constraint method grows sharply with the distance moved between samples. This is illustrated in figure 7. The bias in $t_{x}$ is small though, compared to the distance moved over the sampling interval.

\subsubsection{The Effect of Violation of the Trajectory Constraint on Scene Segmentation}

The trajectory constrained method yields very accurate estimates of the motion even when applied to single static data pairs. This property can be exploited to segment moving objects from a static background. It is necessary to investigate the effect a non circular trajectory has upon scene segmentation.

The background data, consisting of 20 points, were generated as before. The moving object was generated using a 3D Gaussian generator, typically centred between $2000-2500 \mathrm{~mm}$ in front of the vehicle. The object was represented by 10 points. Given the movement of the object, a corresponding set was obtained with respect to the vehicle's new position. A linked list was then produced including both the background and the object points. To illustrate how the segmentation was achieved, only one vehicle movement was recorded.

In the first experiment the vehicle moved along a clothoid of sharpness $k=2 \times 10^{-6}$. The motion parameters were $\theta=5.73^{\circ}, t_{x}=9.90 \mathrm{~mm}$ and $t_{z}=249.8 \mathrm{~mm}$. The object moved $100 \mathrm{~mm}$ horizontally with respect to the position of the vehicle at time $t$. The estimates of of $t_{x}$ and $t_{z}$ obtained by applying the trajectory constrained method to each individual data pair can be seen in figure 8 . It is clear that a group of the points lie in a straight line.

The clustering algorithm described in [4] was applied to these estimates with $\theta_{t h}=0.03^{\circ}$ and $L_{t h}=5$. All 20 of the stationary points in the scene formed a cluster, and the trajectory constraint method was used to estimate the motion parameters using these alone.

The data is clustered on the basis that the variance of the parameter $\theta$ is small. An experiment was carried out to investigate how the variance of the estimates of $\theta$ from individual data points was affected by increased violation of the trajectory consraint. The standard deviation plotted against sharpness coefficient can be seen in figure 9 . The standard deviation does not seem to be increased greatly by violation of the trajectory constraint. So, for reasonable trajectories successful segmentation can be achieved.

\section{Conclusions}

A slight bias is inherent in the trajectory constraint method when the vehicle is not following a circular path as equation (2) is no longer strictly valid. It is however, extremely small for reasonable sampling rates.

Segmentation was only very mildly affected by violation of the trajectory constraint. For clothoids of reasonable sharpness, segmentation is successful, and so very accurate estimates of the motion parameters can still be obtained in the presence of moving objects. 


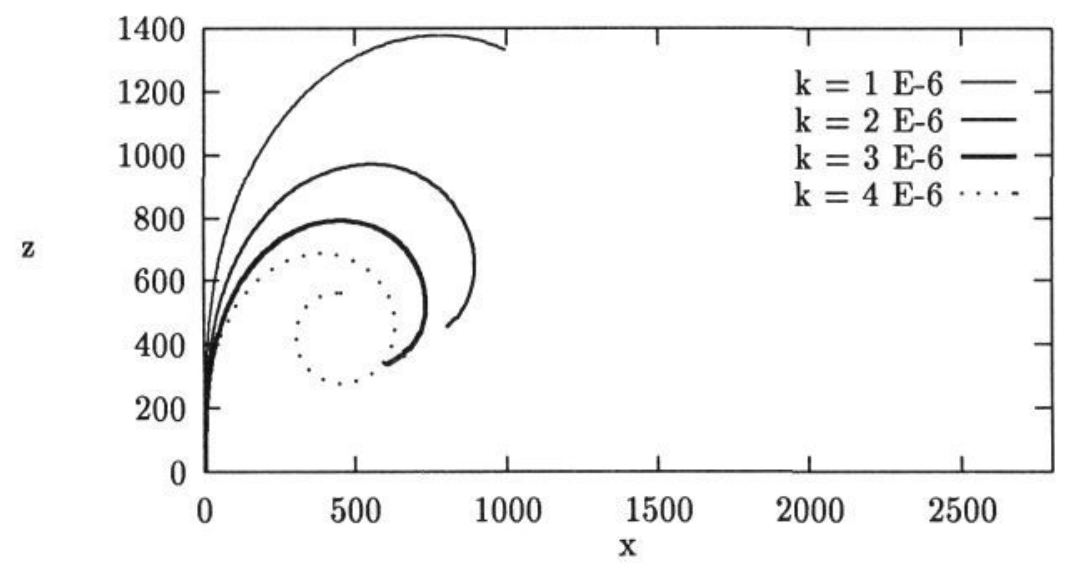

Figure 1: A Set of Clothoids

\section{Acknowledgements}

Dr Y. Zheng is supported by a grant from RSRE awarded to Prof. J. Frisby, Prof. S Billings and Prof J. Mayhew. The authors wish to acknowledge their debt to all the members of AIVRU.

\section{References}

[1] C. Harris and M. Stephens. A combined corner and edge detector. In Proceedings of the Fourth Alvey Vision Conference, pages 147-151, August 1988.

[2] Y. Kanayama and N. Miyake. Trajectory generation for mobile robots. In Robotics Research, volume 3, pages 333-340, 1986.

[3] L. Matthies and T. Kanade. The cycle of uncertainty and constraint in robot perception. In Robotics Research, The Fourth International Symposium, pages $327-335,1988$.

[4] Y. Zheng N.A. Thacker S.A. Billings J.E.W. Mayhew and J.P. Frisby. 3d scene segmentation using a constrained motion parameter recovery algorithm. Technical report, 1991.

[5] Y. Zheng N.A. Thacker S.A. Billings J.E.W. Mayhew and J.P. Frisby. Ground plane motion parameter recovery and $3 \mathrm{~d}$ segmentation using vehicle trajectory constraints. Technical report, 1991.

[6] N.A. Thacker. Corner detection and matching. Memo 58, AIVRU, 1990. 


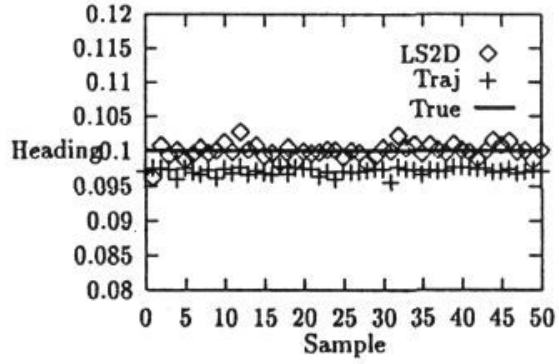

Figure 2: Estimates of $\theta$ in radians for Least Squares and Trajectory Methods for Motion Along a Clothoid

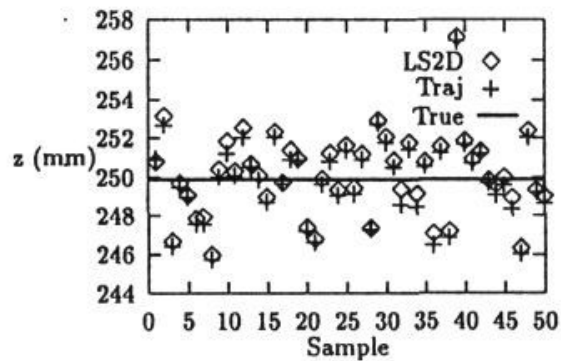

Figure 3: Estimates of $t_{2}(\mathrm{~mm})$ for Least Squares and Trajectory Methods for Motion Along a Clothoid

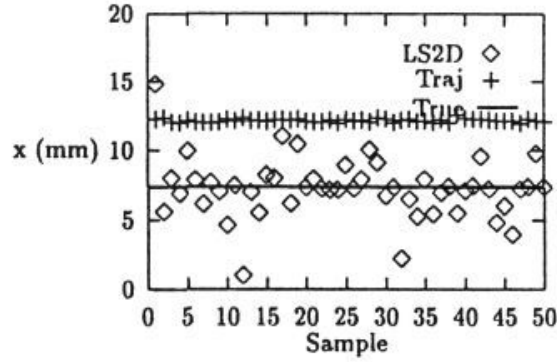

Figure 4: Estimates of $t_{x}(\mathrm{~mm})$ for Least Squares and Trajectory Methods for Motion Along a Clothoid

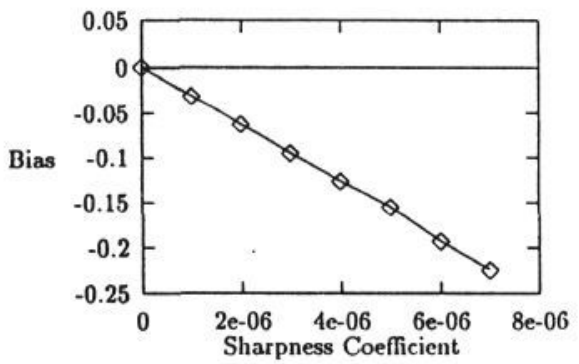

Figure 5: Bias in $\theta(\mathrm{deg})$ for the Trajectory Method for Motion Along Clothoids of Increasing Sharpness 


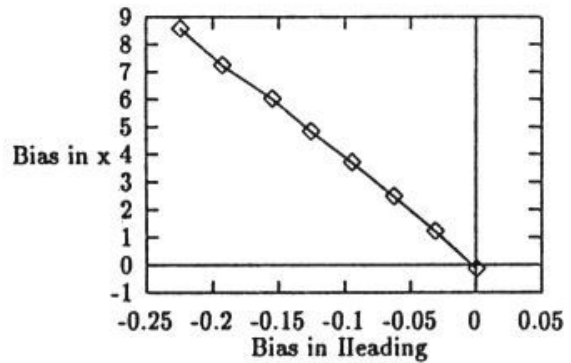

Figure 6: Bias in $t_{x}(\mathrm{~mm})$ against Bias in $\theta(\mathrm{deg})$ for Figure 8: Estimates of $t_{x}$ and $t_{z}$ from Individual Data the Trajectory Method

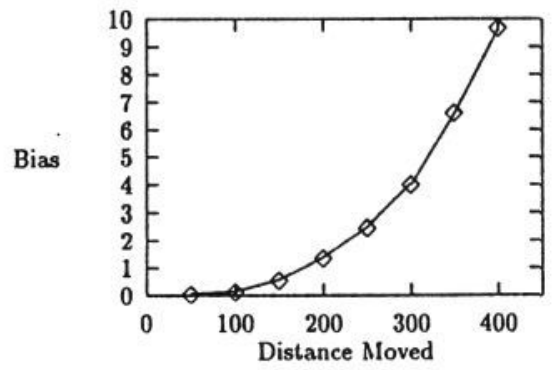

Figure 7: Bias in $t_{s}(\mathrm{~mm})$ for the Trajectory Method Figure 9: Standard Deviation of $\theta$ for Motion along for Different Sampling Rates

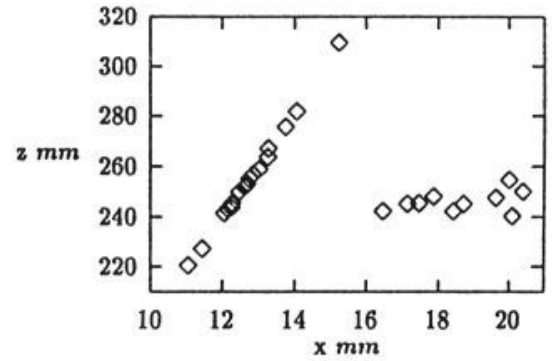

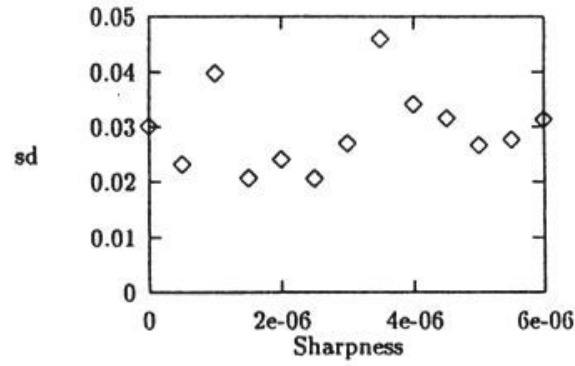

Clothoids of Increasing Sharpness 